\title{
De novo triplication of 11 q12.3 in a patient with developmental delay and distinctive facial features
}

Toshiyuki Yamamoto ${ }^{1 *}$, Mari Matsuo ${ }^{2}$, Shino Shimada ${ }^{1,3}$, Noriko Sangu ${ }^{1,4}$, Keiko Shimojima ${ }^{1}$, Seijiro Aso ${ }^{5}$ and Kayoko Saito ${ }^{2}$

\begin{abstract}
Background: Triplication is a rare chromosomal anomaly. We identified a de novo triplication of 11q12.3 in a patient with developmental delay, distinctive facial features, and others. In the present study, we discuss the mechanism of triplications that are not embedded within duplications and potential genes which may contribute to the phenotype.

Results: The identified triplication of 11q12.3 was $557 \mathrm{~kb}$ long and not embedded within the duplicated regions. The aberrant region was overlapped with the segment reported to be duplicated in 2 other patients. The common phenotypic features in the present patient and the previously reported patient were brain developmental delay, finger abnormalities (including arachnodactuly, camptodactyly, brachydactyly, clinodactyly, and broad thumbs), and preauricular pits.

Conclusions: Triplications that are not embedded within duplicated regions are rare and sometimes observed as the consequence of non-allelic homologous recombination. The de novo triplication identified in the present study is novel and not embedded within the duplicated region. In the 11q12.3 region, many copy number variations were observed in the database. This may be the trigger of this rare triplication. Because the shortest region of overlap contained 2 candidate genes, STX5 and CHRM1, which show some relevance to neuronal functions, we believe that the genomic copy number gains of these genes may be responsible for the neurological features seen in these patients.
\end{abstract}

Keywords: Triplication, STX5, CHRM1, 11q12.3, Developmental delay

\section{Background}

Triplication is a rare chromosomal aberration. Many chromosomal triplications that are visible by conventional karyotyping have been reported. Such chromosomal rearrangements are considered to be caused by abnormal meiotic chromosomal recombinations $[1,2]$. The introduction of chromosomal microarray testing allowed the identification of invisible small triplications. Although we have performed chromosomal microarray testing of $>1,000$ samples in our institution, only 3 invisible triplications have been identified to date (Figure 1). Thus, the frequency of

\footnotetext{
* Correspondence: yamamoto.toshiyuki@twmu.ac.jp

${ }^{1}$ Tokyo Women's Medical University Institute for Integrated Medical Sciences, 8-1 Kawada-cho, Shinjuku-ward, Tokyo 162-8666, Japan

Full list of author information is available at the end of the article
}

such triplications is $<0.3 \%$ in patients with developmental delay and/or congenital abnormalities.

When we analyzed genomic copy numbers in cases of triplication, the patterns of the results could be classified into 2 types according to genomic structures: (1) embedded within the duplicated segments and (2) not embedded within the duplicated segments (Figure 1). Two of the 3 triplications in our laboratory were embedded within the duplicated regions. A triplication identified at 17p13.3, including the platelet-activating factor acetylhydrolase $1 \mathrm{~b}$ regulatory subunit 1 gene (PAFAH1B1) (a gene responsible for lissencephaly) was among them [3] (Figure 1). The triplicated segment was embedded within the duplicated regions, and fiber-fluorescence in situ hybridization (FISH) analysis confirmed that the triplicated segments were in a

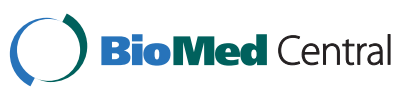



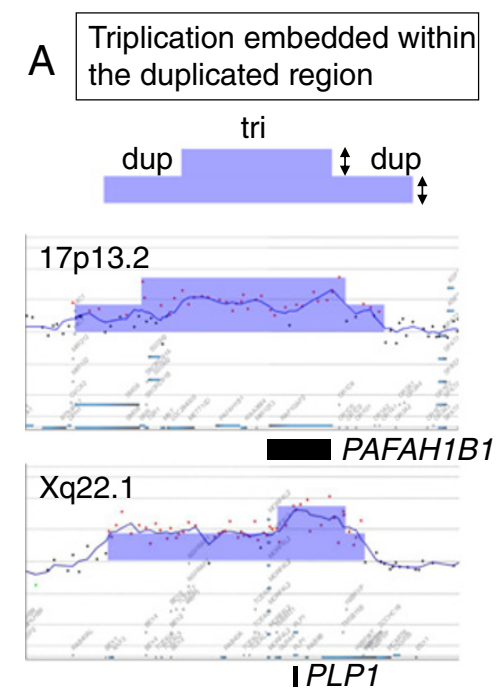
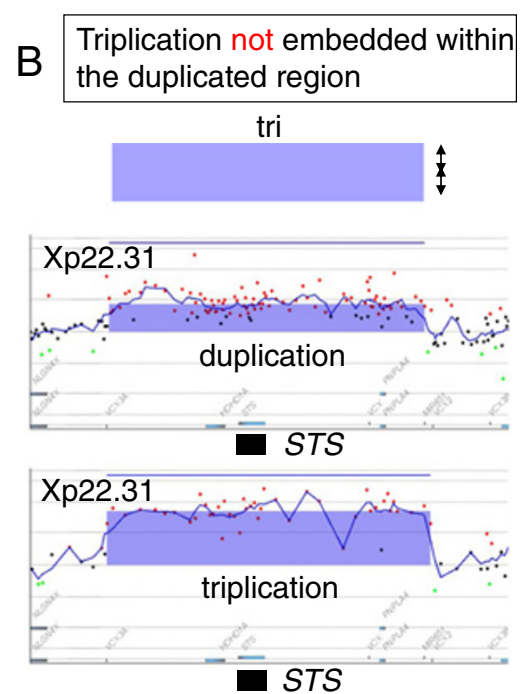

Figure 1 Explanation of the 2 triplication patterns. Each triplication pattern shown by Gene View of Agilent Genomic Workbench (Agilent Technologies) is schematically represented. The blue rectangles indicate chromosomal aberrations. The X-and Y-axes indicate the genomic location and a $\log _{2}$ ratio of the intensity, respectively. The $\log _{2}$ ratio of the triplicated region is 2 -fold higher than that of the duplicated region. (A) The triplication embedded within the duplication region shows duplicated regions (dup) in both ends of the triplication (tri). The 2 examples at 17p13.2 and Xq22.1 include PAFAH1B1 and PLP1, respectively. (B) The triplication not embedded within the duplicated region does not show any duplicated regions on either end of the triplication (tri) Two examples of duplication and triplication at Xp22.31 that include STS are shown. Both aberrations show the same range of the aberration. There are no duplicated regions around the triplicated segment. The black rectangles indicate the locations of the genes.

tandem orientation. A similar triplication of this region was also reported by $\mathrm{Bi}$ et al.; however, the nucleotide sequence of the breakpoints and/or inserted orientation was not analyzed [4]. Although breakpoint junctions have been analyzed in many cases with chromosomal rearrangements [5], it is challenging to map and sequence them in cases of copy number gains due to the existence of extra copies of the fragments, particularly in the case of autosomal chromosomes [6].

Another triplication embedded within the duplicated segments was identified in a patient with PelizaeusMerzbacher disease [7] (Figure 1), whose triplication, including the proteolipid protein 1 gene (PLP1), was caused by the mechanism of duplication-inverted triplicationduplication (DUP-TRP/INV-DUP). This mechanism was first reported by Carvalho et al. as the common mechanism in patients with recurrent triplication around the methyl CpG binding protein 2 gene (MECP2) located on Xq28 [8].

The third triplication identified in the Xp22.31 region was not embedded within the duplicated segments, and the aberration region was same as the duplication that is well-known as a popular copy number variation (CNV) (Figure 1). This triplication, including the steroid sylfatase gene (STS), was previously reported as not being embedded within the duplicated segments because the breakpoints were common among aberrations identified in individuals
[6]. This finding indicated that non-allelic homologous recombination (NAHR) is mediated by low copy repeats (LCRs) surrounding the triplicated structures in this region, the orientation of the inserted triplicated segments remain unknown in those cases.

As mentioned above, a triplication embedded within a duplication is common in cases of random occurrences; however, triplications that are not embedded within duplicated regions may be caused by surrounding LCRs as a consequence of NAHR. In this study, we identified a de novo triplication that was not embedded within a duplication, in a patient with developmental delay and distinctive features. Herein, we discuss the genotypephenotype correlation as well as the mechanism of chromosomal triplication.

\section{Case report}

A 22-month-old girl was born at 38 weeks of gestation, and she was the third child of healthy parents. Her birth weight was $2,714 \mathrm{~g}$ (10 25 centile), length was $46.5 \mathrm{~cm}$ (10 25 centile), and occipitofrontal circumference (OFC) was $34.1 \mathrm{~cm}$ (75 90 centile). Her father and mother were 37 and 35 years old, respectively. At birth, low-set ears were noted. From early infancy, she showed feeding difficulty because of hypotonia and hypersomnia. Early development was delayed, with head control at 5 
months, rolling over at 8 months, sitting at 11 months, crawling at 13 months, and standing unsupported at 22 months. Brain magnetic resonance imaging, electroencephalography, and auditory brainstem response did not show any abnormalities.

At present, her height is $82.1 \mathrm{~cm}$ (10 25 centile), weight is $11.2 \mathrm{~kg}$ (50 75 centile), and OFC is $45.4 \mathrm{~cm}$ (10 25 centile). She has distinctive facial features, including midface hypoplasia, a flat nasal bridge, telecanthus, anteverted nares, a small nose, long philtrum, low-set and posteriorly rotated ears associated with hypoplastic ear cups, a right preauricular pit, thin lips, a high arched palate, and micrognathia. Arachnodactyly is also noted. Neurological examination reveals generalized hypotonia and right esotropia (Table 1).

\section{Results}

The conventional G-banding technique showed a normal female karyotype of 46,XX. By use a 60K array, a gain of the genomic copy number was identified on the 11q12.3 region with a mean $\log _{2}$ ratio of 1.132548 , indicating a triplicated segment of $557 \mathrm{~kb}$ with a description of arr

Table 1 Clinical features of the patients $w$ ith $11 \mathrm{q} 12.3$ duplication/triplication

\begin{tabular}{|c|c|c|c|c|}
\hline & & Present patient & Tyson et al. (2005) & Jehee et al. (2007) \\
\hline & & & (Subject 2) & (Patient 1) \\
\hline & & & DECIPHER \#253705 & \\
\hline \multicolumn{5}{|c|}{ Physical } \\
\hline & short stature & - & + & + \\
\hline & microcephaly & - & + & + \\
\hline & scoliosis & - & + & N A \\
\hline & congenital heart defect & - & + & N A \\
\hline & fingers & arachnodactyly & camptodactyly (5th finger) & brachydactyly \\
\hline & & & brachydactyly (5th finger) & clinodactyly (5th finger \\
\hline & & & & broad thumbs \\
\hline
\end{tabular}

Face

\begin{tabular}{|c|c|c|c|}
\hline upslanting palpebral fissure & - & + & - \\
\hline midface hypoplasia & + & NA & - \\
\hline flat nasal bridge & + & NA & - \\
\hline telecantus & + & NA & - \\
\hline anteverted nare & + & NA & - \\
\hline small nose & + & NA & - \\
\hline long philtrum & + & NA & - \\
\hline low-set and posteriorly rotated ears & + & NA & - \\
\hline preauricular pit & right & + & right \\
\hline high arched palate & + & NA & NA \\
\hline thin lips & + & NA & - \\
\hline micrognathia & + & NA & - \\
\hline developmental delay & + & + & + \\
\hline feeding difficulty & + & NA & NA \\
\hline hypotonia & + & + & NA \\
\hline hypersomnia & + & NA & NA \\
\hline static encephalopathy & - & + & NA \\
\hline brain MRI abnormality & - & NA & NA \\
\hline EEG abnormality & - & NA & NA \\
\hline ABR abnormality & - & NA & NA \\
\hline
\end{tabular}




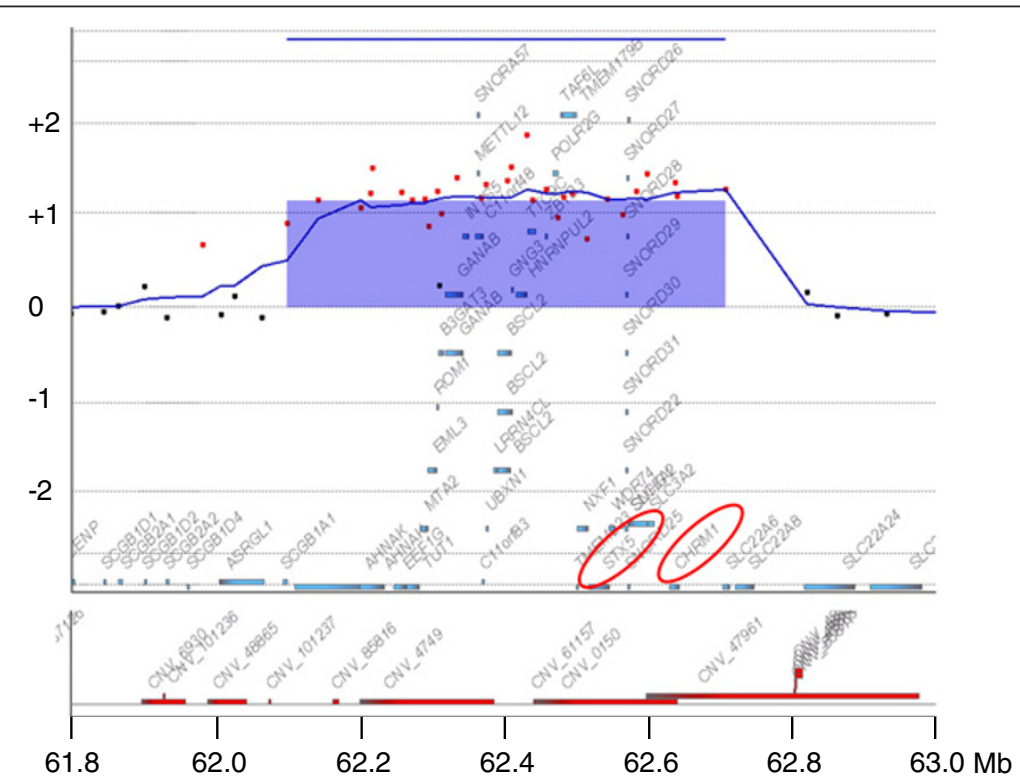

Figure 2 The result of chromosomal microarray testing. (Above) Gene View by Agilent Genomic Workbench (Agilent Technologies) is shown in horizontal view; the $X$ - and $Y$-axes indicate genomic location and a $\log _{2}$ ratio of the intensity, respectively. The region of genomic copy number gain is shown by a blue-translucent rectangle with a mean $\log _{2}$ ratio of 1.132548 with the size of $557 \mathrm{~kb}$ in the $11 \mathrm{q} 12.3$ region. The gene symbols discussed in the text are emphasized by red circles. (Bottom) The locations of the known CNVs are depicted.

11q12.3(62,190,466-62,747,951)x4 (build 19) according to the International System for Human Cytogenetic Nomenclature (2013) (Figure 2). FISH analysis confirmed triplicated signals in the interphase nucleus (Figure 3). One of the targeted signals was stronger than the other on the metaphase spreads of FISH, indicating triplication in 1 of the 2 chromosome 11 homologues. Both parents' samples showed no abnormalities by chromosomal microarray testing using $60 \mathrm{~K}$ (data not shown), indicating de novo occurrence of this triplication.

\section{Discussion}

Compared to the location of the triplication that resulted from DUP-TRP/INV-DUP, the triplicated region identified in the present study was not embedded within the duplicated segments and there was no duplicated region around the triplicated segment (Figure 2). Therefore, the breakpoints caused by this triplication would be the same among all fragments. Such small triplications that are not embedded within duplicated segments are rare, and we were able to identify similar cases in patients with Parkinson's disease associated with triplications in the region of the synuclein alpha gene $(S N C A)$ in the literature [9]. In such cases, triplicated regions, including $S N C A$, are not embedded within duplication. Because the triplicated region identified at 11q12.3 was quite small, we failed to confirm the insertion orientations of the triplicated segments using FISH analysis (data not shown). Due to the existence of many CNVs in this region (Figure 4), it was challenging to confirm the breakpoints of the triplications using polymerase chain reaction-based methods as mentioned in the introduction. Therefore, presently, the insertion orientations of the triplicated segments remain unknown.

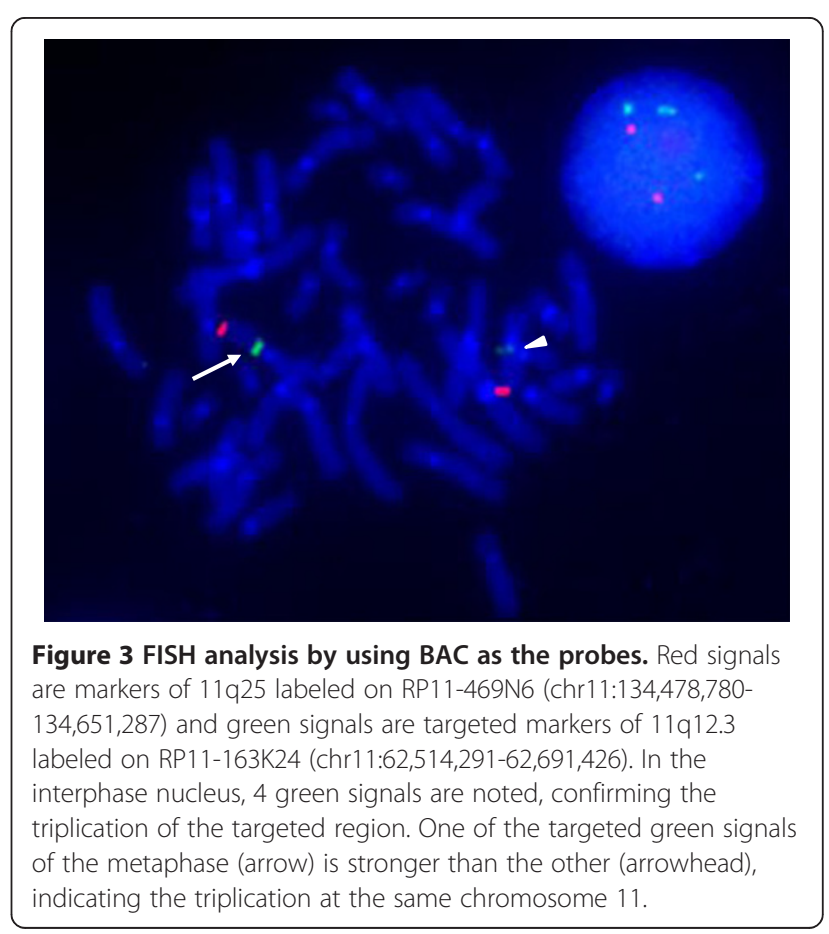




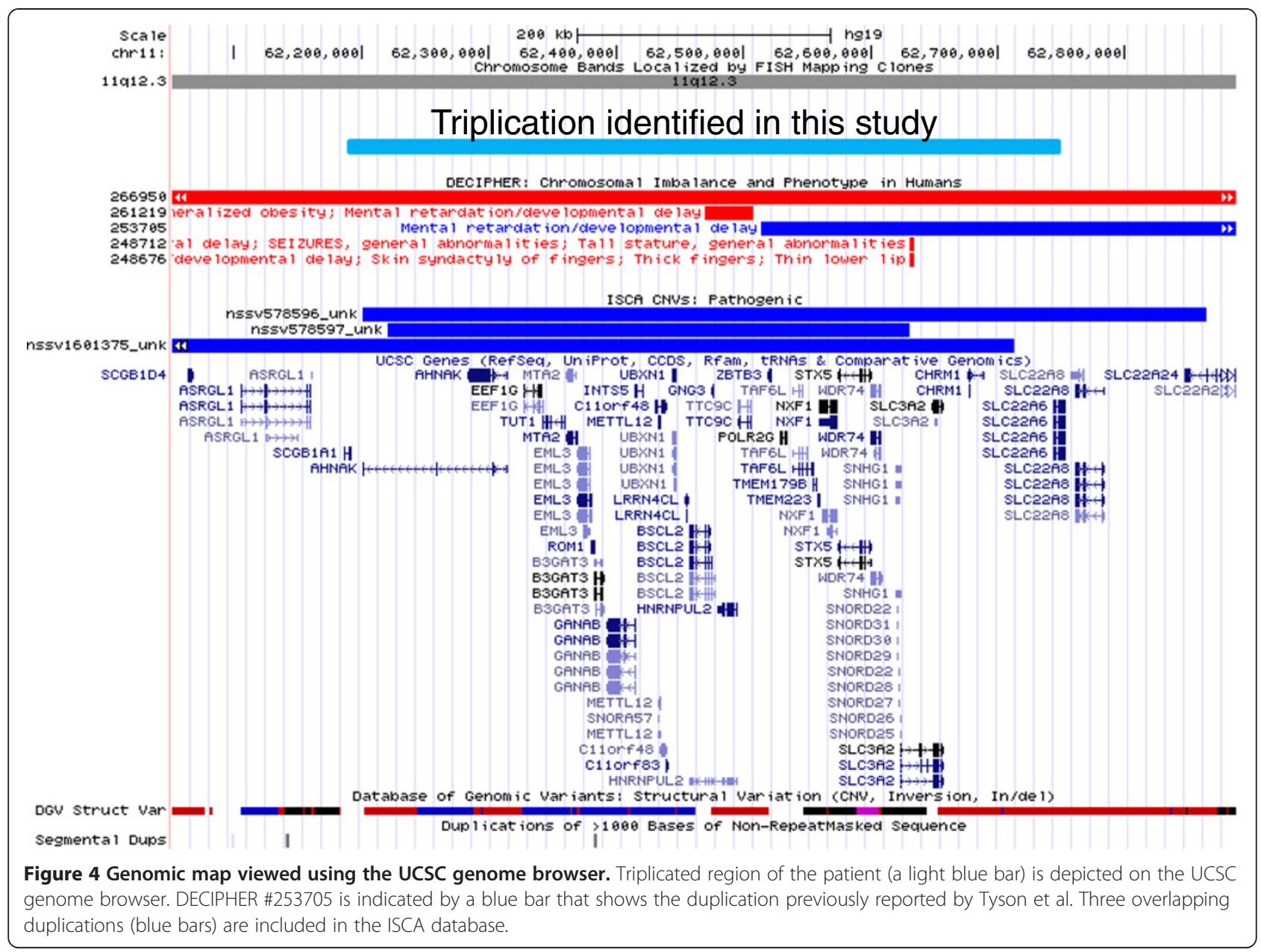

Jehee et al. reported a patient with multiple craniosynostoses, a congenital heart defect, and developmental delay [10]. Cytogenetic analyses identified a mosaic existence of a large duplication of 11q11-q13.3 in that case. Because the duplicated region included the fibroblast growth factor 3 gene (FGF3) and fibroblast growth factor 4 gene (FGF4), Jehee et al. discussed the possibility that the gene dosage effects of these genes resulted in the onset of craniosynostosis [10]. Although the triplicated region identified in the present study was

Table 2 The genes he genes included in the SRO

\begin{tabular}{lll}
\hline & Gene Symbol & Function \\
\hline 1 & ZBTB3 & zinc finger and BTB dom ain containing 3 \\
2 & POLR2G & polymerase (RNA) II (DNA directed) polypeptide G \\
3 & TAF6L & TAF6-like RNA polym erase II, p300/C BP-associated factor (PCAF)-associated factor, 65kD a \\
4 & TMEM179B & transmembrane protein 179B \\
5 & TMEM223 & transmembrane protein 223 \\
6 & NXF1 & nuclear RNA export factor 1 \\
7 & STX5 & syntaxin 5 \\
8 & WDR74 & WD repeat dom ain 74 \\
9 & SNHG1 & small nucleolar RNA host gene 1 (non-protein coding) \\
10 & SLC3A2 & solute carrier family 3 (activators of dibasic and neutral amino acid transport), member 2 \\
11 & CHRM1 & cholinergic receptor muscarinic 1 \\
12 & SLC22A6 & solute carrier family 22 (organic anion transporter), member 6 \\
\hline
\end{tabular}


included in the duplicated region reported by Jehee et al. [10], the present patient did not show any symptoms of craniosynostosis and the candidate genes, FGF3 and FGF4, were not included in the triplicated region because of the extremely small size of the aberrant region.

When we analyzed the International Standard for Cytogenomic Arrays (ISCA) database (https://www. iscaconsortium.org/), we found that 3 duplications were included in this region (Figure 4). Because the ISCA database includes no clinical information, we were unable to compare the phenotypic features of our patient with those of others; however, the existence of similar duplications in this region would suggest that the region may be prone to rearrangement as a result of its structural nature. The DECIPHER database (http://decipher.sanger.ac.uk/) showed only 1 overlapping duplication (chr11:62,514,284-65,140,178) in the 11q12.3 region in a patient (DECIPHER \#253705). This patient had mild-to-moderate intellectual disability and other complications that had already been reported by Tyson et al. [11]. The common phenotypic features between our patient and the previously reported patients include brain developmental delay, finger abnormalities (including arachnodactuly, camptodactyly, brachydactyly, clinodactyly, and broad thumbs), and preauricular pits $[10,11]$ (Table 1). Therefore, we believe that the genes responsible for brain developmental delay and the connective tissue abnormalities are located within the shortest region of overlap (SRO) (Table 2). With regard to the genes responsible for neurological features, including developmental delay and hypotonia, Tyson et al. listed the reticulon 3 gene (RTN3) as the candidate gene because of its highest level of expression in the brain [11]. However, RTN3 is not included within the triplicated region in the present patient.

Alternatively, the syntaxin 5 gene (STX5) and the cholinergic receptor muscarinic 1 gene (CHRM1) (Table 2), in the SRO may be related to the neurological features of these 2 patients because of their functional relevance to the central nervous system. STX5 encodes a member of the syntaxin or t-SNARE (target-SNAP receptor) family [12]. These proteins are found on cell membranes and serve as targets for v-SNAREs (vesicle-SNAP receptors), permitting specific synaptic vesicle docking and fusion [13,14]. CHRM1 is reported to have some relevance to neuronal functions. Muscarinic receptors regulate several effects of acetylcholine in the central and peripheral nervous system. The results of CHRM1null mutant mice investigations suggested that the M1 receptor is specifically involved in memory processes for which the cortex and hippocampus interact [15]. Additionally, the functional relevance of CHRM1 in schizophrenia and depressive disorders has been suggested $[16,17]$.
Since the 1998 review that coined and defined the term "genomic disorders" [18], a multitude of genomic disorders caused by genomic rearrangements have been identified [19], and many of them manifest neurological features, including mental impairments, autistic features, and psychiatric disorders [20,21]. This finding is because there are many dosage-sensitive genes related to nervous system functions. Additionally, there is disputable evidence that CNVs can play a role in the pathogenesis of neurodevelopmental and neurodegenerative disorders [22]. In the present study, we proposed potential candidate genes for developmental delay; however, it is difficult to determine which genes contribute to the connective tissue involvement, including distinctive facial findings, finger abnormalities, and pre-auricular tags seen in the patients with genomic copy number gains of 11p12.3.

\section{Methods}

Blood samples were collected upon approval of the ethics committee of our institution. A patient's karyotype was analyzed using the conventional G-banding technique at 400-500 band resolution. Genomic DNA was extracted from blood samples using QIAamp DNA extraction kit (QIAGEN, Hilden, Germany) and was subsequently evaluated. Chromosomal microarray testing was performed using Agilent Human Genome microarray 60K (Agilent Technologies, Santa Clara, CA), as described previously [23]. Extracted data was analyzed using Agilent Genomic Workbench ver. 6.5 (Agilent Technologies). Metaphase spreads prepared using the patients' blood samples were used for FISH analyses to confirm the results of chromosomal microarray testing. The bacterial artificial clones (BAC), RP11-469N6 and RP11163K24, were selected from the UCSC genome browser (http://genome.ucsc.edu/) for use as probes. Both parental samples were obtained and analyzed using chromosomal microarray testing with a $60 \mathrm{~K}$ array to confirm whether the aberration identified on the patient was de novo.

\section{Consent}

Written informed consent was obtained from the patient's family.

Competing interests

None of the authors has any conflict of interest to disclose.

\section{Authors' contributions}

TY constructed this research, analyzed and interpreted chromosome microarray data, considered the genotype-phenotype correlation, and drafted the paper. SS, NS, and KSh performed the cytogenetic studies. MM and SA correlated clinical findings. KSa supervised and reviewed this article. All authors read and approved the final manuscript.

\section{Acknowledgements}

We would like to express our gratitude to the patient and her family for their cooperation. We also would like to acknowledge Associate Professor Evica Rajcan-Separovic, Department of Pathology (Cytogenetics), Children's and Women's Health Centre of BC, 4480 Oak Street, Vancouver, Canada and the 
DECIPHER database for bringing us the information. This work was partially supported by a Grant-in-Aid for Scientific Research on Innovative Areas "Foundation of Synapse and Neurocircuit Pathology" from the Ministry of Education, Culture, Sports, Science and Technology (MEXT) (TY); a Grant-in-Aid for Scientific Research from Health Labor Sciences Research Grants from the Ministry of Health, Labor, and Welfare, Japan (TY); and a Grant-in-Aid for Young Scientists (B) from Japan Society for the Promotion of Science (JSPS) (KS).

\section{Author details}

${ }^{1}$ Tokyo Women's Medical University Institute for Integrated Medical Sciences, 8-1 Kawada-cho, Shinjuku-ward, Tokyo 162-8666, Japan. ${ }^{2}$ Institute of Medical Genetics, Tokyo Women's Medical University, Tokyo, Japan. ${ }^{3}$ Department of Pediatrics, Tokyo Women's Medical University, Tokyo, Japan. ${ }^{4}$ Department of Oral and Maxillofacial Surgery, School of Medicine, Tokyo Women's Medical University, Tokyo, Japan. ${ }^{5}$ Department of Pediatrics, Japanese Red Cross Medical Center, Tokyo, Japan.

Received: 22 January 2013 Accepted: 1 March 2013 Published: 3 April 2013

\section{References}

1. Reddy KS, Logan JJ: Intrachromosomal triplications: molecular cytogenetic and clinical studies. Clin Genet 2000, 58(2):134-141.

2. Wang J, Reddy KS, Wang E, Halderman L, Morgan BL, Lachman RS, Lin HJ, Cornford ME: Intrachromosomal triplication of 2q11.2-q21 in a severely malformed infant: case report and review of triplications and their possible mechanism. Am J Med Genet 1999, 82(4):312-317.

3. Shimojima K, Sugiura C, Takahashi H, Ikegami M, Takahashi Y, Ohno K, Matsuo M, Saito K, Yamamoto T: Genomic copy number variations at 17p13.3 and epileptogenesis. Epilepsy Res 2010, 89(2-3):303-309.

4. Bi W, Sapir T, Shchelochkov OA, Zhang F, Withers MA, Hunter JV, Levy T, Shinder V, Peiffer DA, Gunderson KL, et al: Increased LIS1 expression affects human and mouse brain development. Nat Genet 2009, 41(2):168-177.

5. Shimojima K, Inoue T, Imai Y, Arai Y, Komoike $Y$, Sugawara M, Fujita T, Ideguchi $H$, Yasumoto $S$, Kanno H, et al: Reduced PLP1 expression in induced pluripotent stem cells derived from a Pelizaeus-Merzbacher disease patient with a partial PLP1 duplication. J Hum Genet 2012, 57(9):580-586.

6. Liu P, Erez A, Nagamani SC, Bi W, Carvalho CM, Simmons AD, Wiszniewska J, Fang P, Eng PA, Cooper ML, et al: Copy number gain at Xp22.31 includes complex duplication rearrangements and recurrent triplications. Hum Mol Genet 2011, 20(10):1975-1988.

7. Shimojima K, Mano T, Kashiwagi M, Tanabe T, Sugawara M, Okamoto N, Arai $\mathrm{H}$, Yamamoto T: Pelizaeus-Merzbacher disease caused by a duplicationinverted triplication-duplication in chromosomal segments including the PLP1 region. Eur J Med Genet 2012, 55(6-7):400-403.

8. Carvalho CM, Ramocki MB, Pehlivan D, Franco LM, Gonzaga-Jauregui C, Fang P, McCall A, Pivnick EK, Hines-Dowell S, Seaver LH, et al: Inverted genomic segments and complex triplication rearrangements are mediated by inverted repeats in the human genome. Nat Genet 2011, 43(11):1074-1081.

9. Singleton $A B$, Farrer $M$, Johnson J, Singleton A, Hague S, Kachergus J, Hulihan M, Peuralinna T, Dutra A, Nussbaum R, et al: alpha-Synuclein locus triplication causes Parkinson's disease. Science 2003, 302(5646):841.

10. Jehee FS, Bertola DR, Yelavarthi KK, Krepischi-Santos AC, Kim C, ViannaMorgante AM, Vermeesch JR, Passos-Bueno MR: An 11q11-q13.3 duplication, including FGF3 and FGF4 genes, in a patient with syndromic multiple craniosynostoses. Am. J. Med. Genet. A 2007, 143A(16):1912-1918

11. Tyson C, Harvard C, Locker R, Friedman JM, Langlois S, Lewis ME, Van Allen M, Somerville M, Arbour L, Clarke L, et al: Submicroscopic deletions and duplications in individuals with intellectual disability detected by array-CGH. Am J Med Genet A 2005, 139(3):173-185.

12. Ravichandran $V$, Roche PA: Cloning and identification of human syntaxin 5 as a synaptobrevin/VAMP binding protein. J Mol Neurosci 1997, 8(2):159-161.

13. Bennett MK, Garcia-Arraras JE, Elferink LA, Peterson K, Fleming AM, Hazuka $\mathrm{CD}$, Scheller $\mathrm{RH}$ : The syntaxin family of vesicular transport receptors. Cell 1993, 74(5):863-873.
14. Sudhof TC, Rothman JE: Membrane fusion: grappling with SNARE and SM proteins. Science 2009, 323(5913):474-477.

15. Anagnostaras SG, Murphy GG, Hamilton SE, Mitchell SL, Rahnama NP, Nathanson NM, Silva AJ: Selective cognitive dysfunction in acetylcholine M1 muscarinic receptor mutant mice. Nat Neurosci 2003, 6(1):51-58.

16. Scarr E, Cowie TF, Kanellakis S, Sundram S, Pantelis C, Dean B: Decreased cortical muscarinic receptors define a subgroup of subjects with schizophrenia. Mol Psychiatry 2009, 14(11):1017-1023.

17. Gibbons AS, Scarr E, McLean C, Sundram S, Dean B: Decreased muscarinic receptor binding in the frontal cortex of bipolar disorder and major depressive disorder subjects. J Affect Disord 2009, 116(3):184-191.

18. Lupski JR: Genomic disorders: structural features of the genome can lead to DNA rearrangements and human disease traits. Trends Genet 1998, 14(10):417-422.

19. Gu W, Lupski JR: CNV and nervous system diseases-what's new? Cytogenet Genome Res 2008, 123(1-4):54-64.

20. Shastry BS: Copy number variation and susceptibility to human disorders (Review). Mol Med Report 2009, 2(2):143-147.

21. Kalman B, Vitale E: Structural chromosomal variations in neurological diseases. Neurologist 2009, 15(5):245-253.

22. Lee JA, Lupski JR: Genomic rearrangements and gene copy-number alterations as a cause of nervous system disorders. Neuron 2006, 52(1):103-121

23. Yamamoto T, Shimojima K, Nishizawa T, Matsuo M, Ito M, Imai K: Clinical manifestations of the deletion of Down syndrome critical region including DYRK1A and KCNJ6. Am J Med Genet A 2011, 155A(1):113-119.

doi:10.1186/1755-8166-6-15

Cite this article as: Yamamoto et al.: De novo triplication of 11q12.3 in a patient with developmental delay and distinctive facial features.

Molecular Cytogenetics 2013 6:15.

\section{Submit your next manuscript to BioMed Central and take full advantage of:}

- Convenient online submission

- Thorough peer review

- No space constraints or color figure charges

- Immediate publication on acceptance

- Inclusion in PubMed, CAS, Scopus and Google Scholar

- Research which is freely available for redistribution

Submit your manuscript at www.biomedcentral.com/submit
C) Biomed Central 